\title{
MicroRNA-425 upregulation indicates better prognosis in younger acute myeloid leukemia patients undergoing chemotherapy
}

\author{
JILEI ZHANG ${ }^{1}$, JINLONG SHI $^{2}$, GAOQI ZHANG $^{1}$, XINPEI ZHANG $^{1}$, XINRUI YANG $^{1}$, \\ SIYUAN YANG ${ }^{1}$, JING WANG ${ }^{1}$, KAI HU ${ }^{1}$, XIAOYAN KE $^{1}$ and LIN FU ${ }^{1}$ \\ ${ }^{1}$ Department of Hematology and Lymphoma Research Center, Peking University, Third Hospital, Beijing 100191; \\ ${ }^{2}$ Department of Biomedical Engineering, Chinese PLA General Hospital, Beijing 100853, P.R. China
}

Received February 6, 2018; Accepted January 24, 2019

DOI: $10.3892 / \mathrm{ol} .2019 .10217$

\begin{abstract}
The aim of the present study was to investigate whether the expression levels of microRNA-425 (miR-425) were associated with the prognosis of acute myeloid leukemia (AML) in patients treated with chemotherapy or allogeneic hematopoietic stem cell transplantation (allo-HSCT). A total of 162 AML patients were enrolled and divided into chemotherapy and allo-HSCT groups. Next, the overall survival (OS) and event-free survival (EFS) were compared between patients with high and low miR-425 expression in each of the treatment groups. In the chemotherapy group, high miR-425 expression was favorable for EFS $(\mathrm{P}=0.001)$ and $\mathrm{OS}(\mathrm{P}=0.001)$ in younger patients ( $<60$ years), whereas it had no effect on EFS and OS in older patients ( $\geq 60$ years). In the allo-HSCT group, there was no association between miR-425 expression levels and clinical outcomes. Further analyses suggested that in the low miR-425 expression group, EFS and OS were longer in patients treated with allo-HSCT as compared with those treated with chemotherapy (both $\mathrm{P}<0.001$ ), whereas no significant differences were observed in the high miR-425 expression group. In conclusion, the current data indicated that miR-425 is an independent favorable prognostic factor for younger AML patients undergoing chemotherapy, and its use may facilitate clinical decision-making in selecting treatment for AML patients. Patients with low miR-425 expression may benefit from allo-HSCT, whereas allo-HSCT did not appear to be beneficial in patients with high miR-425 expression.
\end{abstract}

Correspondence to: Dr Lin Fu, Department of Hematology and Lymphoma Research Center, Peking University, Third Hospital, 49 Huayuan North Road, Beijing 100191, P.R. China E-mail: fulin022@126.com

Key words: microRNA-425, acute myeloid leukemia, chemotherapy, allogeneic hematopoietic stem cell transplantation, prognosis

\section{Introduction}

Acute myeloid leukemia (AML) is the most common type of acute leukemia affecting adults as a complex, dynamic disease (1). AML patients have a highly heterogeneous disease course, and the clinical outcomes are based on cytogenetic abnormalities and molecular genetic aberrations (2,3). For instance, NPMI and biallelic CEBPA mutations are favorable prognostic markers for patients with cytogenetically normal AML (4). In addition, mutations of FLT3-ITD (5), DNMT3A (6), TP53 (7), RUNX1 (8) and MLL-PTD (9) consistently confer poor prognosis in AML patients. Recently, microRNAs (miRNAs) have been reported to serve an important role in the initiation, progression and prognosis of AML as epigenetic alterations (10).

miRNAs are evolutionary conserved, small (typically 18-25 nucleotides), non-coding RNAs that negatively regulate gene expression at the post-transcriptional level and play a crucial part in carcinogenesis. miRNAs not only function as tumor suppressors or oncogenes, but have also been implicated in cell migration and metastasis $(11,12)$. In AML, changes in the expression of several miRNAs have been demonstrated to have functional relevance in leukemogenesis and supply prognostic information, complementing the evidence gained from cytogenetics, gene mutations and altered gene expression (10). For instance, it has been reported that overexpression of miR-191 and miR-199a contributes to an inferior outcome in AML (13), whereas increased expression of miR-181a and miR-212 predicts improved survival rates for AML patients $(14,15)$.

The function of miR-425 has recently been reported in multiple human cancer types (16-19). Previous research has revealed that miR-425 functions as an oncogene or tumor suppressor in different cancer contexts. For instance, miR-425 upregulation promoted cell proliferation, migration and invasion in gastric cancer through a process involving CYLD (20), while it was also reported to inhibit cancer progression in melanoma via IGF-1 (21). However, the potential prognostic role and clinical implications of miR-425 in AML remain unclear.

The present study investigated whether the expression levels of miR-425 provide prognostic information for younger 


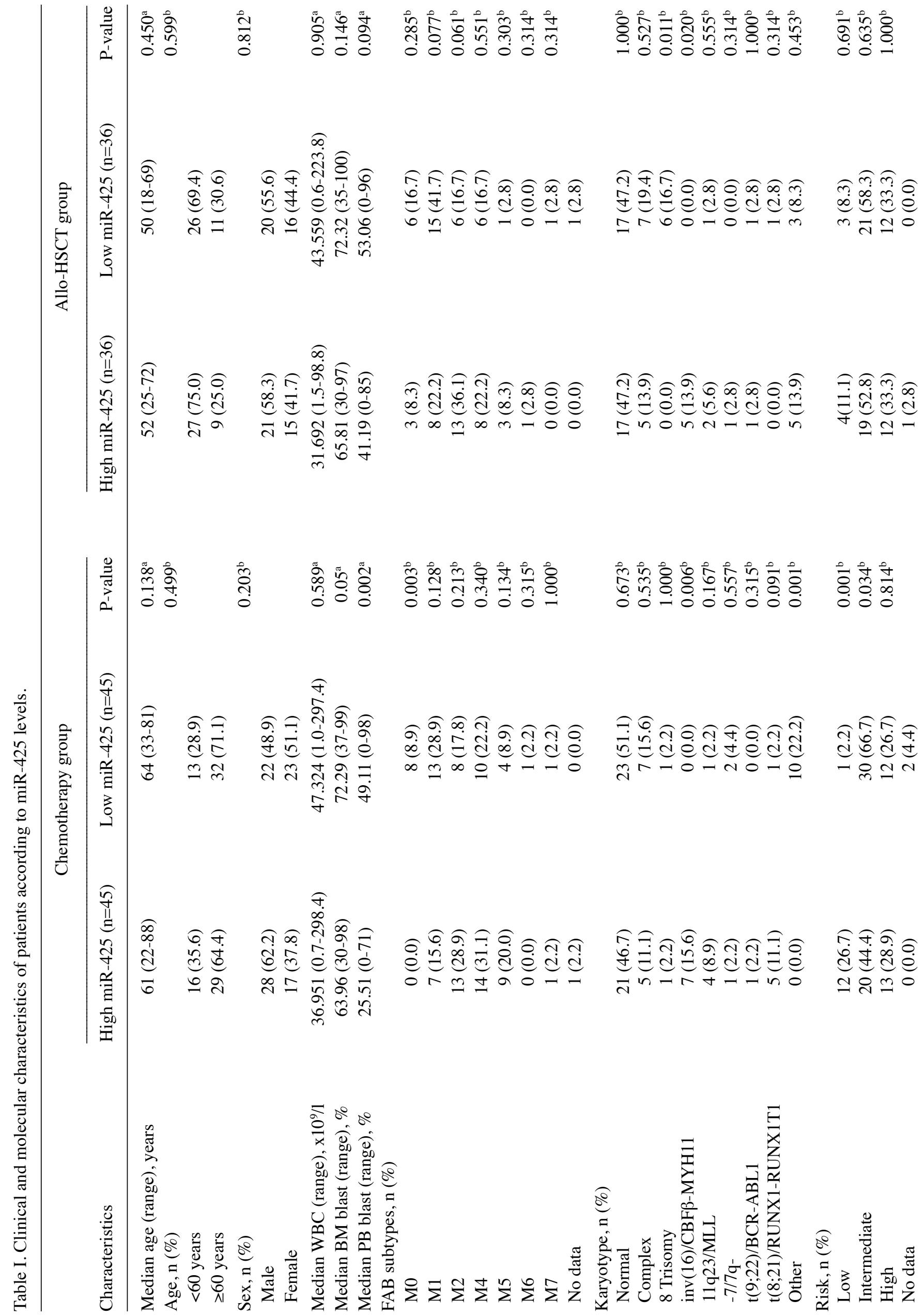




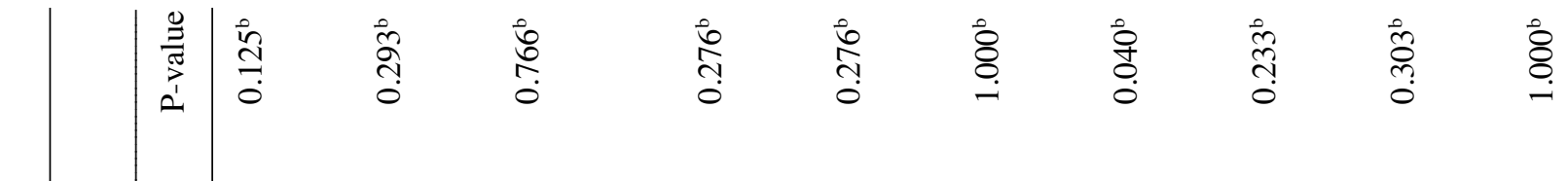

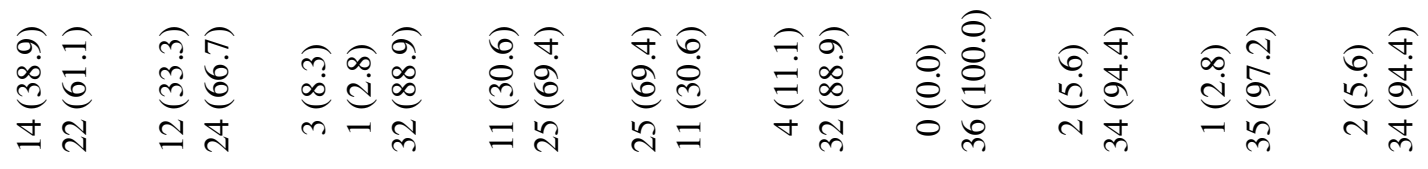

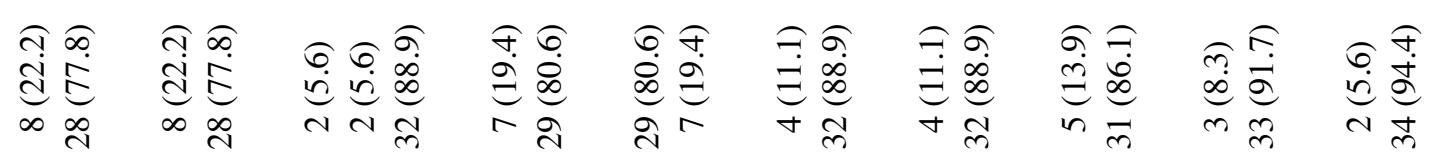

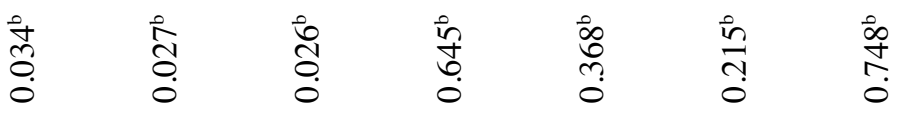

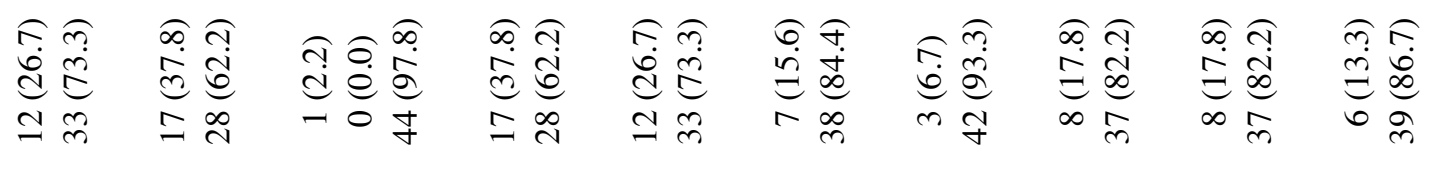

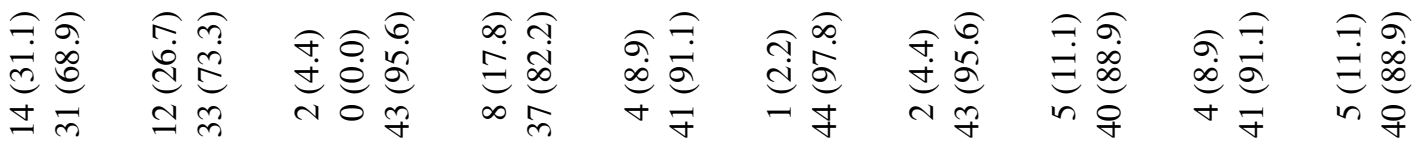

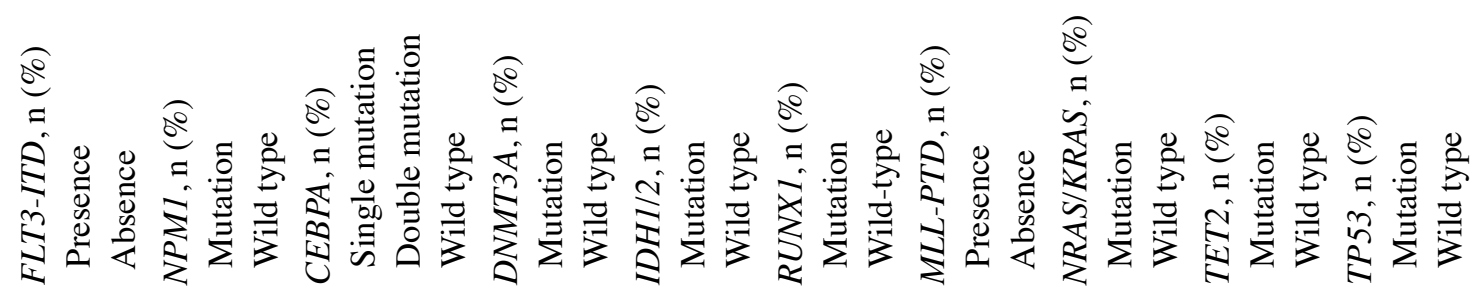


AML patients treated with chemotherapy, independently from a comprehensive panel of other established clinical and molecular predictors. The findings indicated that miR- 425 may have future applications in guiding therapeutic interventions.

\section{Patients and methods}

Patients. The study included a total of 162 AML patients, whose information was retrieved from The Cancer Genome Atlas (TCGA) database (https://cancergenome.nih.gov/). The expression levels of miRNA-425, and the clinical and molecular information of the patients were publicly available from the TCGA website (22). Among the 162 patients, 90 were received chemotherapy-based consolidation as they were treated according to their respective situation, while the remaining 72 patients were treated with allogeneic hematopoietic stem cell transplantation (allo-HSCT). Gene and miRNA expression profiling was performed using HGU133 Plus 2.0 oligonucleotide microarrays (Affymetrix; Thermo Fisher Scientific, Inc., Waltham, MA, USA) and custom miRNA microarrays at diagnosis. Event-free survival (EFS) and overall survival (OS) were considered as endpoints, respectively. EFS was defined as the time from diagnosis until mortality, relapse or the absence of complete remission. OS was determined as the time from diagnosis to mortality, or the end of the follow-up.

Statistical analysis. The clinical and molecular characteristics of patients were summarized using descriptive statistics. Mann-Whitney U test was performed to compare differences in continuous variables, while Pearson's $\chi^{2}$ analysis was utilized to compare the differences in categorical variables. Kaplan-Meier survival curves and the log-rank test were used to compare patient survival. Cox proportional hazards model was used to evaluate miR-425 expression level as a predictor of clinical outcome in the context of other prognostic factors in univariate and multivariate analyses. These other prognostic factors included white blood cell (WBC) count $\left(<20 \times 10^{9} / 1\right.$ vs. $\geq 20 \times 10^{9} / 1$ ), age ( $\geq 60$ vs. $<60$ years), and FLT3-ITD, NPM1, DNMT3A, TP53, RUNX1, TET2, CEBPA, MLL-PTD and $I D H 1 / 2$ mutations. Statistical analyses were performed with SPSS (version 22.0; IBM Corp, Armonk, NY, USA) and GraphPad Prism (version 7.0; GraphPad Software, Inc., La Jolla, CA, USA) software. The results in all analyses were considered as statistically significant when the two-tailed P-value was $<0.05$.

\section{Results}

Association of clinical and molecular characteristics with miR-425 expression levels in the chemotherapy and allo-HSCT groups. A total of 162 AML patients were divided into the chemotherapy and allo-HSCT groups. Next, each group was divided into two further subgroups based on the median expression level of miR-425. Patients with miR-425 expression levels that were higher or equal to the median value were included in the high miR-425 expression group, while the remaining patients were included in the low miR-425 expression. The median expression level was 3,709.321 (range, 481.232-19,682.91) in the chemotherapy group and 3,171.966 
Table II. Univariate and multivariate analyses for EFS and OS in the chemotherapy group.

A, Univariate analysis

\begin{tabular}{|c|c|c|c|c|}
\hline \multirow[b]{2}{*}{ Variables } & \multicolumn{2}{|l|}{ EFS } & \multicolumn{2}{|l|}{ OS } \\
\hline & $\operatorname{HR}(95 \% \mathrm{CI})$ & P-value & $\operatorname{HR}(95 \% \mathrm{CI})$ & P-value \\
\hline miR-425 (high vs. low) & $0.466(0.289-0.750)$ & 0.002 & $0.506(0.316-0.811)$ & 0.005 \\
\hline Age ( $\geq 60$ vs. $<60$ years $)$ & $3.588(2.005-6.421)$ & $<0.001$ & $3.423(1.919-6.106)$ & $<0.001$ \\
\hline WBC (<20 vs. $\left.\geq 20 \times 10^{9} / 1\right)$ & $0.964(0.608-1.528)$ & 0.876 & $0.936(0.591-1.484)$ & 0.779 \\
\hline FLT3-ITD & $1.181(0.715-1.951)$ & 0.517 & $1.168(0.707-1.931)$ & 0.544 \\
\hline$N P M 1$ mutation & $0.893(0.547-1.456)$ & 0.649 & $0.958(0.587-1.562)$ & 0.862 \\
\hline DNMT3A mutation & $1.407(0.852-2.322)$ & 0.182 & $1.432(0.868-2.362)$ & 0.160 \\
\hline TP53 mutation & $2.949(1.510-5.761)$ & 0.002 & $2.898(1.487-5.649)$ & 0.002 \\
\hline$R U N X 1$ mutation & $1.464(0.700-3.064)$ & 0.312 & $1.591(0.759-3.335)$ & 0.219 \\
\hline TET2 mutation & $1.049(0.538-2.045)$ & 0.889 & $1.198(0.614-2.337)$ & 0.597 \\
\hline$M L L-P T D$ & $1.177(0.429-3.228)$ & 0.751 & $1.099(0.401-3.013)$ & 0.855 \\
\hline IDH1/2 mutation & $1.198(0.678-2.118)$ & 0.543 & $1.098(0.621-1.941)$ & 0.748 \\
\hline
\end{tabular}

B, Multivariate analysis

$\begin{array}{lcccc}\text { Younger patients (age, }<60 \text { years) } & & & & \\ \text { miR-425 (high vs. low) } & 0.059(0.011-0.323) & 0.001 & 0.040(0.006-0.279) & 0.001 \\ \text { WBC }\left(<20 \text { vs. } \geq 20 \times 10^{9} / 1\right) & 2.032(0.383-10.782) & 0.405 & 1.768(0.367-8530) & 0.478 \\ \text { FLT3-ITD } & 1.604(0.243-10.570) & 0.623 & 1.319(0.199-8.739) & 0.774 \\ \text { NPM1 mutation } & 0.255(0.020-3.305) & 0.296 & 0.159(0.009-2.813) & 0.210 \\ \text { DNMT3A mutation } & 13.826(1.342-142.405) & 0.027 & 23.130(1.657-322.884) & 0.020 \\ \text { TET2 mutation } & 2.195(0.261-18.438) & 0.469 & 4.481(0.441-45.567) \\ \text { IDH1/2 mutation } & 10.116(0.989-103.475) & 0.051 & 15.114(1.076-212.379) & 0.205 \\ \text { Older patients (age, } \geq 60 \text { years) } & & & 0.044 \\ \text { miR-425 (high vs. low) } & 0.631(0.337-1.180) & 0.149 & 0.752(0.411-1.374) & 0.353 \\ \text { WBC }\left(<20 \text { vs. } \geq 20 \times 10^{9} / 1\right) & 1.188(0.608-2.323) & 0.615 & 1.039(0.544-1.987) & 0.907 \\ \text { FLT3-ITD } & 1.149(0.530-2.491) & 0.724 & 1.029(0.470-2.256) & 0.942 \\ \text { NPM1 mutation } & 0.874(0.415-1.837) & 0.722 & 0.981(0.465-2.066) & 0.959 \\ \text { DNMT3A mutation } & 1.013(0.478-2.150) & 0.972 & 1.075(0.521-2.219) & 0.844 \\ \text { TP53 mutation } & 2.216(0.886-5.099) & 0.091 & 1.859(0.798-4.333) & 0.151 \\ \text { RUNX1 mutation } & 1.020(0.373-2.790) & 0.970 & 1.157(0.429-3.124) & 0.773 \\ \text { TET2 mutation } & 1.419(0.571-3.524) & 0.451 & 2.033(0.826-5.004) & 0.123 \\ \text { MLL-PTD } & 1.300(0.386-4.382) & 0.672 & 1.892(0.551-6.494) & 0.311 \\ I D H 1 / 2 \text { mutation } & 1.388(0.608-3.171) & 0.436 & 1.536(0.673-3.507) & 0.308\end{array}$

EFS, event-free survival; OS, overall survival; HR, hazard ratio; 95\% CI, 95\% confidence interval; miR, microRNA; WBC, white blood cell.

(range, 942.01-17,575.09) in the allo-HSCT group. The correlation of the miR-425 expression level with the clinical and molecular characteristics of patients is fully described in Table I.

In the chemotherapy group, patients with high miR-425 expression level exhibited a higher prevalence of inv(16)/CBF $\beta$ MYH11 and low-risk disease, whereas the percentage of peripheral blood (PB) blasts, French-American-British (FAB) classification subtype M0, intermediate-risk disease, and DNMT3A, IDHI/2 and RUNX1 mutations were lower in these patients. There were no significant differences between the two expression groups in terms of age and gender distribution,
WBCs, bone marrow (BM) blasts, FAB subtypes other than M0, karyotypes other than inv(16)/CBF $\beta-M Y H 11$, low-risk disease, relapse rate, and FLT3-ITD, NPM1, CEBPA, MLL-PTD, NRAS/KRAS, TET2 and TP53 mutations.

In the allo-HSCT group, patients with high miR-425 expression level exhibited increased prevalence of inv(16)/CBF $\beta$-MYH11 and $M L L-P T D$ mutations, whereas the prevalence of trisomy 8 karyotype was lower in these patients. There were no significant differences between the two expression groups in terms of age and gender distribution, WBCs, BM blasts, PB blasts, FAB classification, risk stratification, frequent AML mutations (FLT3-ITD, NPM1, CEBPA, 
Table III. Univariate and multivariate analyses for EFS and OS in the allogeneic hematopoietic stem cell transplantation group.

A, Univariate analysis

\begin{tabular}{|c|c|c|c|c|}
\hline \multirow[b]{2}{*}{ Variables } & \multicolumn{2}{|c|}{ EFS } & \multicolumn{2}{|l|}{ OS } \\
\hline & HR $(95 \% \mathrm{CI})$ & $\mathrm{P}$-value & $\mathrm{HR}(95 \% \mathrm{CI})$ & P-value \\
\hline miR-425 (high vs. low) & $0.983(0.576-1.678)$ & 0.951 & $0.932(0.544-1.598)$ & 0.798 \\
\hline Age $(\geq 60$ vs. $<60$ years $)$ & $1.003(0.748-1.345)$ & 0.982 & $1.397(0.777-2.512)$ & 0.265 \\
\hline WBC (<20 vs. $\left.\geq 20 \times 10^{9} / 1\right)$ & $1.244(0.726-2.132)$ & 0.426 & $1.052(0.614-1.806)$ & 0.851 \\
\hline FLT3-ITD & $1.242(0.690-2.236)$ & 0.469 & $1.244(0.692-2.235)$ & 0.466 \\
\hline NPM1 mutation & $0.864(0.470-1.590)$ & 0.639 & $0.879(0.478-1.617)$ & 0.678 \\
\hline DNMT3A mutation & $1.141(0.619-2.104)$ & 0.672 & $1.269(0.686-2.347)$ & 0.447 \\
\hline TP53 mutation & $1.750(0.623-4.912)$ & 0.288 & $3.788(1.289-11.133)$ & 0.015 \\
\hline RUNX1 mutation & $1.545(0.725-3.290)$ & 0.260 & $2.523(1.046-4.849)$ & 0.038 \\
\hline TET2 mutation & $1.270(0.708-2.278)$ & 0.423 & $1.099(0.614-1.969)$ & 0.750 \\
\hline$C E P B A$ double mutation & $0.603(0.145-2.517)$ & 0.488 & $0.616(0.149-2.539)$ & 0.502 \\
\hline$M L L-P T D$ & $6.529(2.185-19.511)$ & 0.001 & $3.106(1.104-8.741)$ & 0.032 \\
\hline IDH1/2 mutation & $1.192(0.863-1.646)$ & 0.287 & $1.117(0.810-1.540)$ & 0.500 \\
\hline
\end{tabular}

B, Multivariate analysis

\begin{tabular}{|c|c|c|c|c|}
\hline miR-425 (high vs. low) & $0.983(0.594-1.917)$ & 0.960 & $0.764(0.404-1.444)$ & 0.408 \\
\hline Age $(\geq 60$ vs. $<60$ years $)$ & $1.179(0.588-2.364)$ & 0.643 & $1.458(0.736-2.888)$ & 0.280 \\
\hline WBC $\left(<20\right.$ vs. $\left.\geq 20 \times 10^{9} / 1\right)$ & $1.515(0.773-2.972)$ & 0.227 & $1.134(0.584-2.200)$ & 0.711 \\
\hline FLT3-ITD & $1.251(0.552-2.834)$ & 0.591 & $1.626(0.717-3.690)$ & 0.245 \\
\hline NPM1 mutation & $0.858(0.368-2.004)$ & 0.724 & $0.908(0.370-2.230)$ & 0.834 \\
\hline DNMT3A mutation & $1.248(0.599-2.598)$ & 0.554 & $1.567(0.740-3.318)$ & 0.240 \\
\hline TP53 mutation & $2.273(0.692-7.466)$ & 0.176 & $5.271(1.549-17.938)$ & 0.008 \\
\hline$R U N X 1$ mutation & $1.661(0.670-4.118)$ & 0.273 & 3.039 (1.181-7.817) & 0.021 \\
\hline TET2 mutation & $1.812(0.509-6.443)$ & 0.359 & $1.968(0.505-7.675)$ & 0.329 \\
\hline$C E P B A$ double mutation & $0.531(0.116-2.434)$ & 0.415 & $0.717(0.161-3.198)$ & 0.663 \\
\hline$M L L-P T D$ & $4.713(1.234-18.002)$ & 0.023 & $1.884(0.532-6.667)$ & 0.326 \\
\hline IDHI/2 mutation & $1.332(0.582-3.047)$ & 0.498 & $1.429(0.617-3.311)$ & 0.405 \\
\hline
\end{tabular}

EFS, event-free survival; OS, overall survival; HR, hazard ratio; 95\% CI, 95\% confidence interval; miR, microRNA; WBC, white blood cell.

DNMT3A, IDH1/2, RUNX1, NRAS/KRAS, TET2 and TP53) and relapse rate.

Univariate and multivariate Cox analysis for prognosis in the chemotherapy and allo-HSCT groups. The effect of clinical and molecular characteristics on survival was next evaluated. The results of this analysis for the chemotherapy and allo-HSCT groups are summarized in Tables II and III.

In the chemotherapy group (Table II), univariate analysis revealed that high miR-425 expression was associated with significantly more favorable EFS $(\mathrm{P}=0.002)$ and OS $(\mathrm{P}=0.005)$, while TP53 mutations were associated with poor EFS $(\mathrm{P}=0.002)$ and $\mathrm{OS}(\mathrm{P}=0.002)$. Since the age group was observed to be a significant predictive factor for EFS and OS (all $\mathrm{P}<0.001$ ), younger and older subgroups were analyzed separately in multivariate analyses. In younger patients, only FLT3-ITD, NPM1, DNMT3A, TET2, IDH1/2 was included in multivariate analysis due to their relatively high mutation rate, which is more than $5 \%$ in younger patients. The results indicated that high miR-425 expression independently predicted a longer EFS and OS (both $\mathrm{P}=0.001$ ). However, DNMT3A mutation in younger patients indicated a relatively shorter EFS $(\mathrm{P}=0.027)$ and $\mathrm{OS}(\mathrm{P}=0.020)$, and $I D H 1 / 2$ mutation also indicated shorter OS $(\mathrm{P}=0.044)$. In older patients, the miR-425 expression level was not associated with survival.

In the allo-HSCT group, univariate and multivariate analyses indicated that TP53 $(\mathrm{P}=0.015$ and 0.008 , respectively) and RUNX1 ( $\mathrm{P}=0.038$ and 0.021 , respectively) mutations contributed to poor OS. In addition, $M L L-P T D$ mutations had an adverse effect on EFS and OS in univariate analysis (both $\mathrm{P}<0.05)$, and remained significantly associated with shorter EFS in multivariate analysis $(\mathrm{P}=0.023)$. However, miR-425 had no effect on EFS and OS in univariate and multivariate analyses.

Subsequently, AML patients in both the chemotherapy and allo-HSCT groups were also analyzed as a whole using multivariate analysis, and the results are presented in Table IV. 
Table IV. Multivariate analysis for EFS and OS in all patients.

\begin{tabular}{|c|c|c|c|c|}
\hline \multirow[b]{2}{*}{ Variables } & \multicolumn{2}{|c|}{ EFS } & \multicolumn{2}{|l|}{$\mathrm{OS}$} \\
\hline & $\mathrm{HR}(95 \% \mathrm{CI})$ & P-value & HR & P-value \\
\hline miR-425 (high vs. low) & $0.713(0.473-1.074)$ & 0.106 & $0.814(0.543-1.220)$ & 0.319 \\
\hline Age ( $\geq 60$ vs. $<60$ years $)$ & $2.065(1.374-3.105)$ & $<0.001$ & $2.218(1.458-3.373)$ & $<0.001$ \\
\hline WBC ( $<20$ vs. $\left.\geq 20 \times 10^{9} / 1\right)$ & $1.365(0.900-2.070)$ & 0.143 & $1.144(0.760-1.723)$ & 0.519 \\
\hline Treatment (chemo vs. allo-HSCT) & $0.653(0.443-0.963)$ & 0.031 & $0.549(0.370-0.817)$ & 0.003 \\
\hline FLT3-ITD & $1.262(0.783-2.035)$ & 0.339 & $1.299(0.793-2.128)$ & 0.298 \\
\hline NPM1 mutation & $0.932(0.570-1.523)$ & 0.778 & $0.958(0.581-1.580)$ & 0.867 \\
\hline DNMT3A mutation & $1.455(0.946-2.236)$ & 0.087 & $1.591(1.044-2.423)$ & 0.031 \\
\hline TP53 mutation & $2.735(1.401-5.340)$ & 0.003 & $3.307(1.675-6.526)$ & 0.001 \\
\hline$R U N X 1$ mutation & $1.754(0.962-3.196)$ & 0.067 & $2.170(1.185-3.974)$ & 0.012 \\
\hline TET2 mutation & $1.306(0.691-2.469)$ & 0.410 & $1.584(0.853-2.942)$ & 0.145 \\
\hline$C E P B A$ double mutation & $0.890(0.206-3.856)$ & 0.877 & $0.981(0.228-4.214)$ & 0.979 \\
\hline$M L L-P T D$ & $1.999(0.903-4.426)$ & 0.088 & $1.900(0.868-4.159)$ & 0.108 \\
\hline IDHI/2 mutation & $1.503(0.907-2.493)$ & 0.114 & $1.437(0.873-2.365)$ & 0.154 \\
\hline
\end{tabular}

EFS, event-free survival; OS, overall survival; HR, hazard ratio; 95\% CI, 95\% confidence interval; miR, microRNA; WBC, white blood cell; chemo, chemotherapy; allo-HSCT, allogeneic hematopoietic stem cell transplantation.
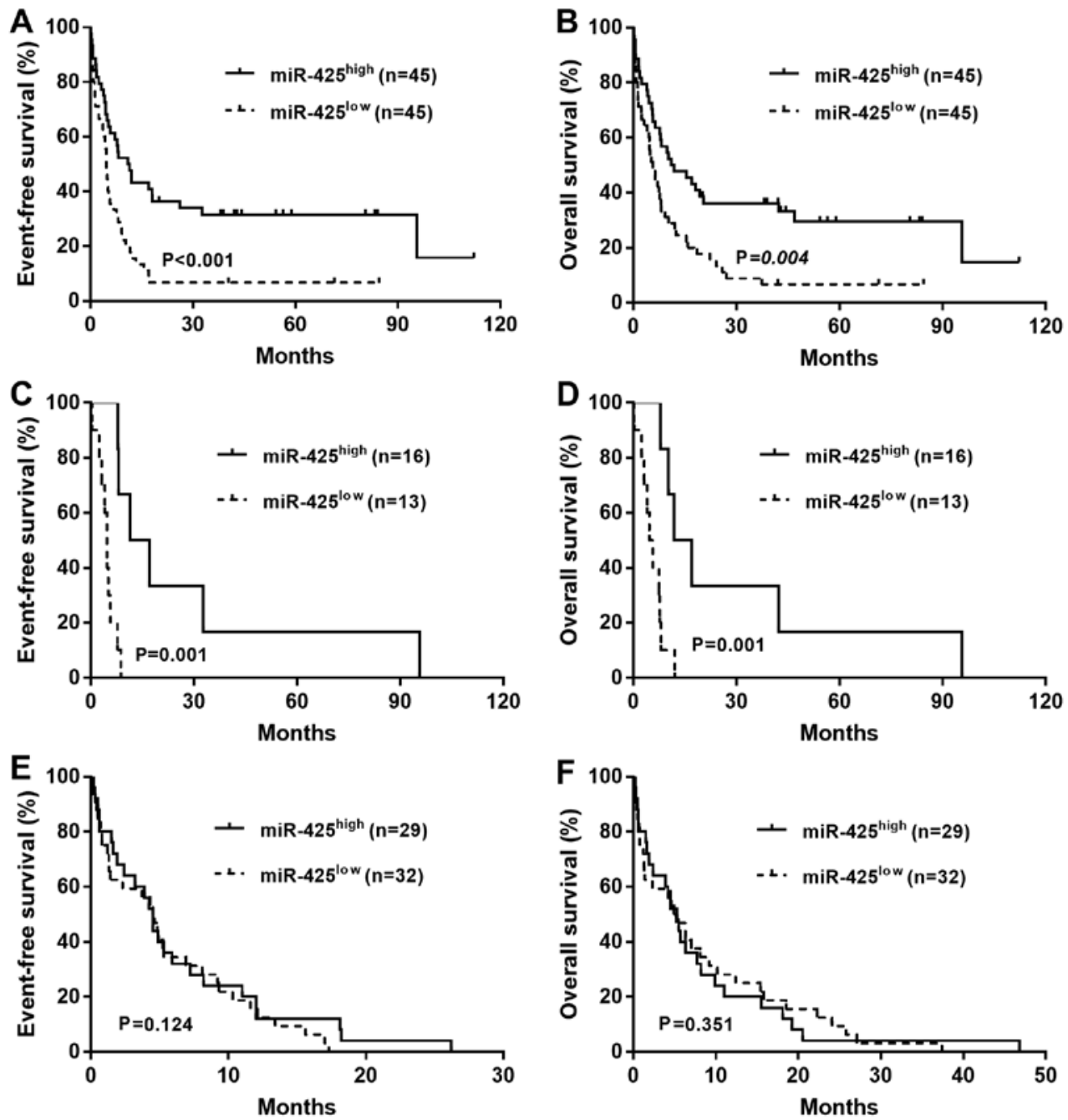

Figure 1. Kaplan-Meier curves of EFS and OS in the chemotherapy and allo-HSCT groups. (A) EFS and (B) OS in the chemotherapy group were longer in patients with high miR-425 expression. (C) EFS and (D) OS in younger patients undergoing chemotherapy were longer in the high miR-425 expression group. (E) EFS and (F) OS in older patients undergoing chemotherapy were similar in the groups with low and high miR-425 expression. 

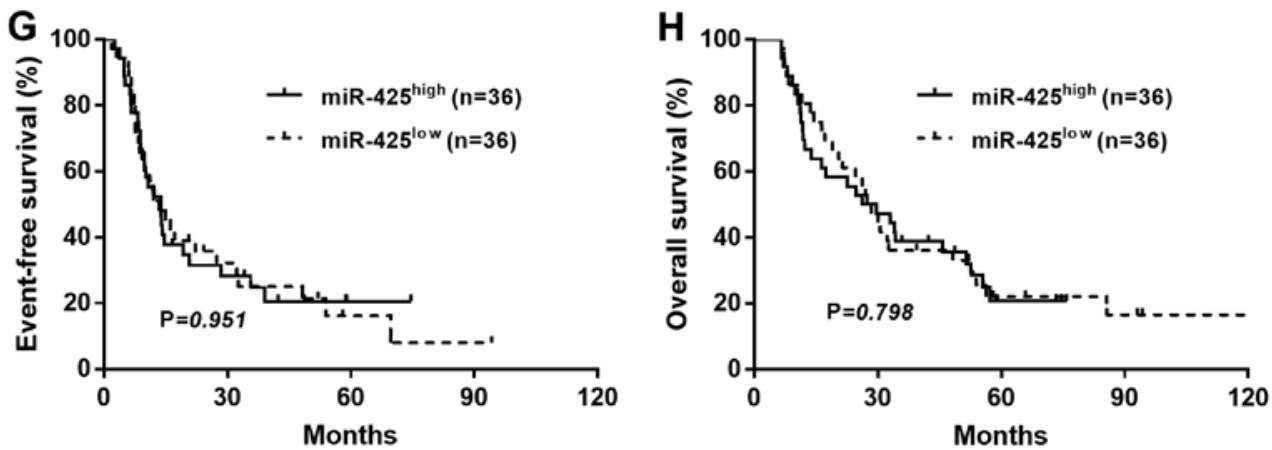

Figure 1. Continued. (G) EFS and (H) OS in the allo-HSCT group were not significantly different between patients with high and low miR-425 expression. EFS, event-free survival; OS, overall survival; allo-HSCT, allogeneic hematopoietic stem cell transplantation; miR, microRNA.
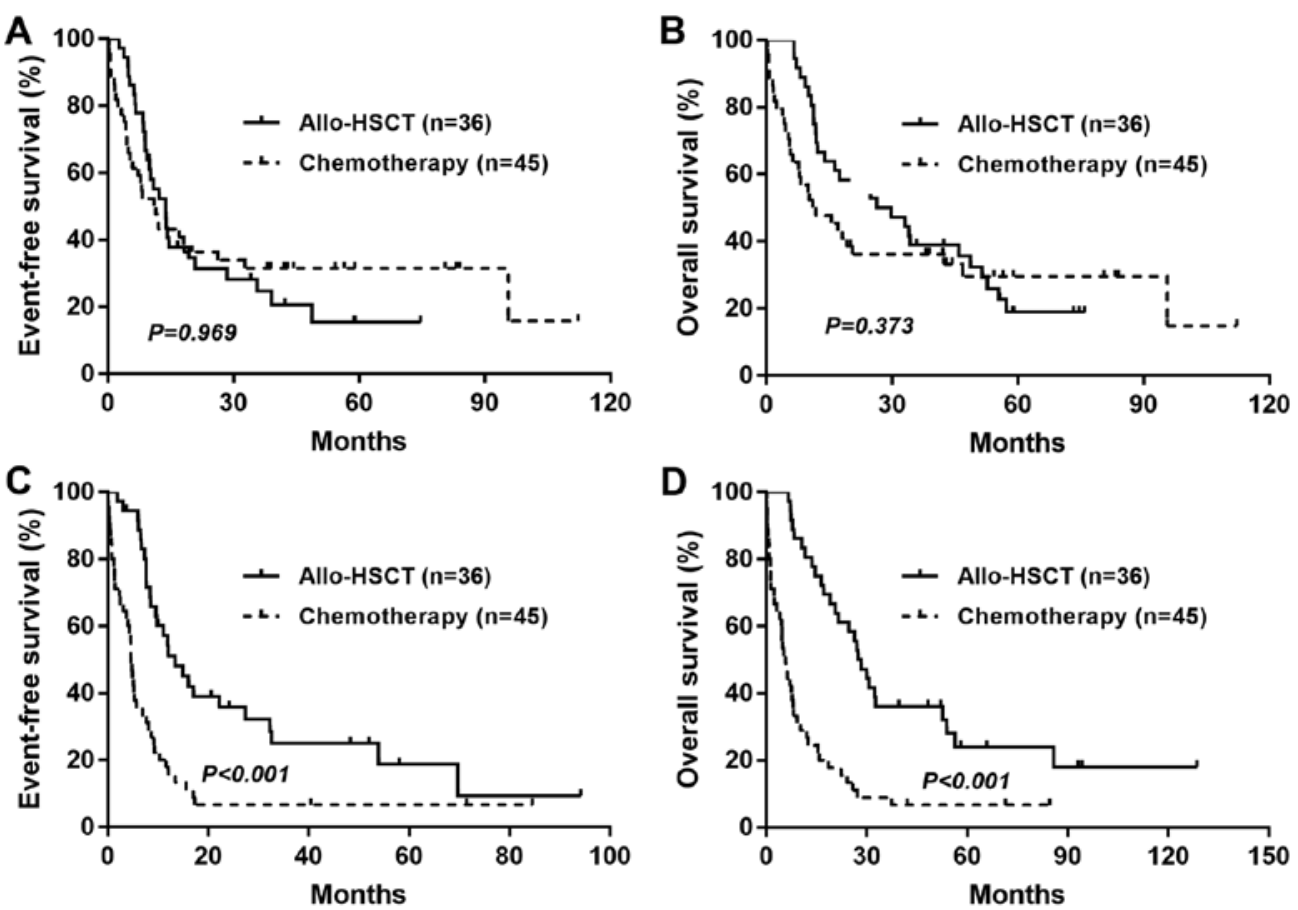

Figure 2. Kaplan-Meier curves of EFS and OS in patients with high and low miR-425 expression. (A) EFS and (B) OS in patients with high miR-425 expression were not significantly different between the allo-HSCT and chemotherapy groups. (C) EFS and (D) OS in the low miR-425 expression group were longer in patients treated with allo-HSCT as compared with those treated with chemotherapy. EFS, event-free survival; OS, overall survival; allo-HSCT, allogeneic hematopoietic stem cell transplantation; miR, microRNA.

Mutations in DNMT3A and RUNX1 were observed to have an unfavorable effect on $\mathrm{OS}(\mathrm{P}=0.031$ and 0.012 , respectively), while older age, chemotherapy and TP53 mutations contributed to poor EFS and OS (all $\mathrm{P}<0.05$ ). No significant differences were identified in EFS and OS between the high and low miR-425 expression groups.

Prognostic value of miR-425 expression. Kaplan-Meier survival estimate in the chemotherapy group indicated a better prognosis for EFS $(\mathrm{P}<0.001)$ and OS $(\mathrm{P}=0.004)$ in patients with high expression of miR-425 as compared with that in patients exhibiting low miR-425 expression (Fig. 1A and B). Upon the division of AML patients undergoing chemotherapy into a younger and older age group, miR-425 was only associated with EFS and OS (both $\mathrm{P}=0.001$ ) in younger patients (Fig. 1C and D), whereas no significant prognostic value was observed in older patients (Fig. $1 \mathrm{E}$ and F). In the
allo-HSCT group, no significant differences were observed between patients with high versus low miR-425 expression (Fig. $1 \mathrm{G}$ and $\mathrm{H}$ ).

Next, the entire cohort of patients was divided into two groups according to the expression levels of miR-425. Kaplan-Meier survival estimate demonstrated that no significant differences were observed between patients treated with allo-HSCT and chemotherapy in the high miR-425 expression group (Fig. 2A and B). By contrast, EFS and OS (both $\mathrm{P}<0.001)$ were longer in patients treated with allo-HSCT as compared with those treated with chemotherapy in the low miR-425 expression group (Fig. 2C and D).

\section{Discussion}

In the current study, higher miR-425 expression indicated better survival prospects for younger AML patients who received 
chemotherapy. By contrast, miR-425 expression exhibited no prognostic value in patients treated with allo-HSCT.

The data reported in the present study revealed that low-risk patients and the favorable cytogenetic alteration inv(16)/CBF $3-$ MYH11 appeared more frequently in the high miR-425 expression group, while unfavorable genetic mutations in $R U N X 1$ were more often observed in the low expression group. This implies that miR-425 upregulation may serve the same role as inv(16)/CBF $\beta-\mathrm{MYH} 11$ in predicting the prognosis for AML patients. Accordingly, downregulation of miR-425 may have similar prognostic features to RUNX1 mutation. Univariate analysis in the chemotherapy group indicated a putative favorable role of high miR-425 expression in AML patients. Furthermore, it was observed that the patient age had considerable implications on the therapeutic outcomes, and high miR-425 expression only indicated longer EFS and OS in younger AML patients that received chemotherapy. Kaplan-Meier survival curve analysis indicated the same results. By contrast, miR-425 expression levels were found to have no effect in the allo-HSCT group, suggesting that allo-HSCT overrides the prognostic ability of miR-425 expression.

Epigenetic modifiers, such as IDH1/2, TET2 and DNMT3A mutations, affect the expression of genes that are crucial to leukemogenesis, and as a consequence, they powerfully influence the prognosis of AML. In addition, IDH1, IDH2 and TET2 mutations are known to modulate DNA hydroxymethylation (23), while DNMT3A mutations are involved in DNA methylation, and increased risk of relapse or mortality in AML (24). It was demonstrated in the present study that the incidence of IDHI/2 and DNMT3A mutations was significantly higher in patients with low miR-425 expression, suggesting that miR-425 may also affect the prognosis through epigenetic regulation.

Allo-HSCT is one of the curative treatment options for patients with AML (25). In order to decide between transplant and non-transplant consolidation strategies, it is crucial to gain a clear idea of the outcome to be expected subsequent to allo-HSCT (26). The present study findings suggested that allo-HSCT may be more effective for AML patients expressing low miR-425 levels, whereas it may not be as effective for patients with high miR-425 expression, thus highlighting the potential utility of miR-425 in treatment selection.

There are certain limitations in the current study. Firstly, when patients treated with chemotherapy were analyzed by age subgroup, the sample size in each age group was small; in particular, there were only 29 patients in the younger subgroup. In addition, certain genes were required to be deleted from the multivariate analysis due to their low mutation rate, in order to ensure statistical efficiency. Finally, although the association between miR-425 expression levels and clinical outcomes was illustrated in this pilot study, further laboratory work is required to elucidate whether miR-425 functions as a tumor suppressor in AML and the underlying mechanisms involved. In our future work, in vitro and in vivo mouse experiments will be conducted to identify the target genes or pathways.

In conclusion, to the best of our knowledge, the present study analysis is the first to demonstrate that high miR-425 expression is an independent positive prognostic factor in younger AML patients undergoing chemotherapy. In addition, miR-425 upregulation may a factor for advising against allo-HSCT in AML patients.

\section{Acknowledgements}

Not applicable.

\section{Funding}

The present study was supported by grants from the National Natural Science Foundation of China (grant nos. 81500118 and 61501519), the China Postdoctoral Science Foundation Funded Project (grant no. 2016M600443) and the PLAGH Project of Medical Big Data (grant no. 2016MBD-025).

\section{Availability of data and materials}

The datasets analyzed during this study are available in The Cancer Genome Atlas database (https://cancergenome.nih.gov/).

\section{Authors' contributions}

LF and XK proposed and designed the study, and XK suggested analysis of the data based on age group of patients who underwent chemotherapy. JS screened and collected the data. XZ and $X Y$ were responsible for quality control of the data and performed the statistical analysis. GZ and JZ analyzed and interpreted the data, and JZ was a major contributor in writing the manuscript. KH performed the analysis, and generated the tables and figures. SY and JW interpreted the data, drafted the discussion, and revised and edited the entire manuscript. All authors read and approved the final manuscript.

\section{Ethics approval and consent to participate}

Not applicable.

\section{Patient consent for publication}

Not applicable.

\section{Competing interests}

The authors declare that they have no competing interests.

\section{References}

1. Papaemmanuil E, Gerstung M, Bullinger L, Gaidzik VI, Paschka P, Roberts ND, Potter NE, Heuser M, Thol F, Bolli N, et al: Genomic classification and prognosis in acute myeloid leukemia. N Engl J Med 374: 2209-2221, 2016.

2. Tsai CH, Hou HA, Tang JL, Liu CY, Lin CC, Chou WC, Tseng MH, Chiang YC, Kuo YY, Liu MC, et al: Genetic alterations and their clinical implications in older patients with acute myeloid leukemia. Leukemia 30: 1485-1492, 2016.

3. Ustun C and Marcucci G: Emerging diagnostic and therapeutic approaches in core binding factor acute myeloid leukaemia. Curr Opin Hematol 22: 85-91, 2015.

4. Dohner H, Estey EH, Amadori S, Appelbaum FR, Büchner T, Burnett AK, Dombret H, Fenaux P, Grimwade D, Larson RA, et al: Diagnosis and management of acute myeloid leukemia in adults: Recommendations from an international expert panel, on behalf of the european leukemianet. Blood 115: 453-474, 2010.

5. Dohner H, Estey E, Grimwade D, Amadori S, Appelbaum FR, Büchner T, Dombret H, Ebert BL, Fenaux P, Larson RA, et al: Diagnosis and management of AML in adults: 2017 ELN recommendations from an international expert panel. Blood 129: 424-447, 2017. 
6. Brunetti L, Gundry MC and Goodell MA: DNMT3A in Leukemia. Cold Spring Harb Perspect Med 7: a030320, 2017

7. Meyer SC and Levine RL: Translational implications of somatic genomics in acute myeloid leukaemia. Lancet Oncol 15: e382-e394, 2014

8. Metzeler KH, Herold T, Rothenberg-Thurley M, Amler S, Sauerland MC, Görlich D, Schneider S, Konstandin NP, Dufour A, Bräundl K, et al: Spectrum and prognostic relevance of driver gene mutations in acute myeloid leukemia. Blood 128: 686-698, 2016.

9. Patel JP, Gonen M, Figueroa ME, Fernandez H, Sun Z, Racevskis J, Van Vlierberghe P, Dolgalev I, Thomas S, Aminova O, et al: Prognostic relevance of integrated genetic profiling in acute myeloid leukemia. N Engl J Med 366: 1079-1089, 2012.

10. Marcucci G, Mrozek K, Radmacher MD, Garzon R and Bloomfield CD: The prognostic and functional role of microRNAs in acute myeloid leukemia. Blood 117: 1121-1129, 2011.

11. Zhou X, Wen W, Shan X, Zhu W, Xu J, Guo R, Cheng W, Wang F, Qi LW, Chen Y, et al: A six-microRNA panel in plasma was identified as a potential biomarker for lung adenocarcinoma diagnosis. Oncotarget 8: 6513-6525, 2017.

12. Garzon R, Marcucci G and Croce CM: Targeting microRNAs in cancer: Rationale, strategies and challenges. Nat Rev Drug Discov 9: 775-789, 2010.

13. Garzon R, Volinia S, Liu CG, Fernandez-Cymering C, Palumbo T, Pichiorri F, Fabbri M, Coombes K, Alder H, Nakamura T, et al: MicroRNA signatures associated with cytogenetics and prognosis in acute myeloid leukemia. Blood 111: 3183-3189, 2008.

14. Schwind S, Maharry K, Radmacher MD, Mrózek K, Holland KB, Margeson D, Whitman SP, Hickey C, Becker H, Metzeler KH, et al: Prognostic significance of expression of a single microRNA, miR-181a, in cytogenetically normal acute myeloid leukemia: A cancer and leukemia group B study. J Clin Oncol 28: 5257-5264, 2010.

15. Sun SM, Rockova V, Bullinger L, Dijkstra MK, Döhner H, Löwenberg B and Jongen-Lavrencic M: The prognostic relevance of miR-212 expression with survival in cytogenetically and molecularly heterogeneous AML. Leukemia 27: 100-106, 2013.

16. Zhang Z, Wen M, Guo J, Shi J, Wang Z, Tan B, Zhang G, Zheng $X$ and Zhang A: Clinical value of miR-425-5p detection and its association with cell proliferation and apoptosis of gastric cancer. Pathol Res Pract 213: 929-937, 2017.
17. Fang F, Song T, Zhang T, Cui Y, Zhang G and Xiong Q: MiR-425-5p promotes invasion and metastasis of hepatocellular carcinoma cells through SCAI-mediated dysregulation of multiple signaling pathways. Oncotarget 8: 31745-31757, 2017.

18. Zhang Y, Hu X, Miao X, Zhu K, Cui S, Meng Q, Sun J and Wang T: MicroRNA-425-5p regulates chemoresistance in colorectal cancer cells via regulation of programmed cell death 10 . J Cell Mol Med 20: 360-369, 2016.

19. Sun L, Jiang R, Li J, Wang B, Ma C, Lv Y and Mu N: MicoRNA-425-5p is a potential prognostic biomarker for cervical cancer. Ann Clin Biochem 54: 127-133, 2017.

20. Yan YF, Gong FM, Wang BS and Zheng W: MiR-425-5p promotes tumor progression via modulation of CYLD in gastric cancer. Eur Rev Med Pharmacol Sci 21: 2130-2136, 2017.

21. Liu P, Hu Y, Ma L, Du M, Xia L and Hu Z: miR-425 inhibits melanoma metastasis through repression of PI3K-Akt pathway by targeting IGF-1. Biomed Pharmacother 75: 51-57, 2015.

22. Cancer Genome Atlas Research Network: Genomic and epigenomic landscapes of adult de novo acute myeloid leukemia. N Engl J Med 368: 2059-2074, 2013.

23. Conway O'Brien E, Prideaux S and Chevassut T: The epigenetic landscape of acute myeloid leukemia. Adv Hematol 2014: 103175, 2014.

24. Marcucci G, Metzeler KH, Schwind S, Becker H, Maharry K, Mrózek K, Radmacher MD, Kohlschmidt J, Nicolet D, Whitman SP, et al: Age-related prognostic impact of different types of DNMT3A mutations in adults with primary cytogenetically normal acute myeloid leukemia. J Clin Oncol 30: 742-750, 2012.

25. Gupta V, Tallman MS and Weisdorf DJ: Allogeneic hematopoietic cell transplantation for adults with acute myeloid leukemia: Myths, controversies, and unknowns. Blood 117: 2307-2318, 2011.

26. Schmid C, Labopin M, Socie G, Daguindau E, Volin L, Huynh A, Bourhis JH, Milpied N, Cornelissen J, Chevallier P, et al: Outcome of patients with distinct molecular genotypes and cytogenetically normal AML after allogeneic transplantation. Blood 126: 2062-2069, 2015. 